\title{
Zur Frage: Ob der physiologische Contractionsact von der Spannung beeinflusst wird?
}

\author{
Von \\ Dr. F. Schenck.
}

(Mit 2 Abbildungen.)

Die Richtigkeit der Lehre, dass der physiologisehe Contractionsact des Muskels von der Spannung beeinflusst wird, ist jüngst von B lix ${ }^{1}$ ) bestritten worden. Er behauptet, die Thatsachen, auf die man bisher jene Lehre gestitzt hat, seien auch erklärlich dureh die Annahme, dass nicht der eigentlich physiologische Contractionsact dureh Spannungsänderung eine Aenderung erleide, sondern, dass die Spannung nur auf rein mechanisehe Folgen des Contractionsacts wirke, nämlich auf elastische Nachwirkungen, innere Versehiebungen der Muskelröhren und Muskelbündel und dergl. Die Gesammtheit dieser secundären Gestaltveränderungen des Muskels will ich mit dem schon von anderen Autoren vor Blix eingefubrten Wort "Nachschrumpfung" kurz bezeichnen. Ausser der Nachschrumpfung sollen ferner noch Verschiedenheiten in der Grösse der inneren Widerstände eine Rolle spielen bei dem Zustandekommen der Erscheinungen, auf die man bisher die alte Lehre gegrindet hat. Der innere Widerstand hemmt jede Formveränderung, jede Bewegung und „das um so stärker, je schneller die Bewegung ist - schwächer also für den ermüdeten Muskel mit seinen trägen Bewegungen, als für den frischen Muskel mit seinen lebhaften Umgestaltungen".

Auf die Bedeutung dieser Widerstände möchte ich zunächst .hier eingehen, weil mir Blix in einer vor kurzem erschienenen Abbandlung ${ }^{2}$ ) den Vorwurf zu machen scheint, dass ich die Be-

1) Skandinav. Archiv f. Physiolog. Bd. V. S. 150 u. Bd. VI. S. 240.

2) Skandinav. Archiv f. Physiolog. Bd. VI. S. 242. 
deutung dieser Widerstände in einem früheren Aufsatze ${ }^{1}$ ), in dem ich mich schon gegen Blix' Hypothese ausgesprochen habe, unberücksichtigt gelassen habe.

Nehmen wir, um die Betrachtung zu vereinfachen, an, die Nachschrumpfung sei nicht an der Contraction betheiligt, dann würden bloss zwei Factoren die Grösse der Verkürzung bedingen:

1) Die Grösse der contractilen Kraft.

2) Die Grösse des inneren Widerstands, die in diesem Falle für ein und denselben Verkiirzungsgrad immer constant wäre. Die contractile Kraft hat sich mit dem inneren Widerstand ins Gleichgewicht $\mathbf{z u}$ setzen. Wenn nun eine bestimmte contractile Kraft momentan im Muskel entsteht, wird nicht auch, momentan die Verkürzung da sein, die dem Gleichgewicht der contractilen Kraft und des Widerstands entspricht, sondern die Verkürzung wird erst einige Zeit nach Entstehen der contractilen Kraft vollständig erreicht sein. Wie gross ist nun die Zeit, die vergeht vom Entstehen der contractilen Kraft bis zu dem Zeitpunkt, wo die maximale Verkürzung erreicht ist? Man wird diese Zeit schätzen können auf Grund einer Angabe, die ich bei von Kries ${ }^{2}$ ) finde. von Kries hat Versuche angestellt, in denen er die Last eines tetanisirten Muskels plötzlich verminderte, eine Maassnalıme, die für unsere Betrachtung einer momentanen Zunabme der contractilen Kräfte gleichbedeutend ist. Er fand, dass die dadurcb bedingte Verkïrzung (abgesehen von dem Zuwachs durch Nachschrumpfung, der sich allmählich noch einstellte) in $1 / 43$ " erreicht war. Diese Zahl duirfte aber noch zu gross sein, weil in den Versuchen von v. Kries der Muskel nicht momentan entlastet wurde, sondern Verzögerung der Entlastung statthatte durch den remanenten Magnetismus des Elektromagneten, mit dem die grosse Last vor der Entlastung an dem Muskelhebel festgehalten war. .

Entwickèlt sich die contractile Kraft nicht momentan, sondern allmählich, so ist genïgend Zeit vorhanden, dass sich in jedem Augenblick die jeweils vorhandene contractile Kraft mit den Widerständen ins Gleichgewicht setzt; die in jedem Zeitpunkt vorhandene Verkürzung ist also bei langsamer Entwicklung der contractilen Kraft gleich derjenigen, welche dem Gleichgewicht

1) Dies Archiv Bd. 59. S. 395.

2) Du B o is - R e y m on d's Archiv 1880. S. 361. 
Zur Frage: Ob d. physiol. Contractionsact von d. Spannung beeinflusst wird. 501

der contractilen Kraft und der Widerstände entspricht. Dasselbe gilt für langsame Entlastung.

Nehmen wir nun an, wir vergleichen eine Zuckung, bei der wir im Anfang den Muskel an der Verkürzung luemmen, so dass er Spannung statt Verkürzung erzengt, und ihn nun in einem bestimmten Zeitpunkt frei lassen, mit einer ganz ungehemmten Zuckung. Bei der Zuckung mit Anfangshemmung fängt die contractile Kraft momentan, in dem Zeitpunkt, wo der Muskel frei gelassen wird, an zu wirken, bei der ungehemmten Zuckung entwickeln sich die contractilen Kräfte langsamer. Deshalb muss die Verkürzung gleich nach der Anfangshemmung geringer sein, als in entsprechender Zeit bei der Zuckung ohne Hemmung, aber schon. spätestens $1 / 40$ " später müsste der Unterschied in der Verkürzung bei beiden Arten verschwunden sein und von da ab der Verlauf der Verkürzung in beiden Fällen der gleiche sein, wenn der physiologische Contractionsact durch die grössere Spannung bei der Zuckung mit Anfangshemmung nicht beeinflusst wïrde und, wie vorhin angenommen wurde, Nachschrumpfung keine Rolle spielt.

Es lässt sich also allgemein über den Einfluss der Widerstände sagen, dass nach einer vorïbergehenden Spannungsvermehrung während der Zuckung die Verkürzung durch kurze Zeit hindurch geringer ist, als bei der Zuckung ohne Spannungsänderung. Die durch die Spannungsänderung bewirkte Verminderung der Verkürzung ist um so geringer, je langsamer die Entspannung erfolgt.

Bei den Erscheinungen, auf die man die Lehre von dem Einfluss der Spannung auf den Contractionsact stiitzt, handelt es sich aber um ganz andere Dinge, als die eben beschriebenen, leicht zu verstehenden Veränderungen des Zuckungsverlaufs, die durch die Widerstände allein bedingt sind. Aus diesem Grunde bin ich in der früheren Abhandlung nicht auf die Bedeutung der Widerstände eingegangen. Die Widerstände scheinen mir ganz bedeutungslos für die Erklärung der in Betracht kommenden Erscheinungen. Da Blix nun aber verlangt, dass ihre Wirkung berïcksichtigt werde, so will ich im Folgenden da, wo es nöthig ist, doch darauf eingehen.

Es muss nun freilich noch darauf aufmerksam gemacht werden, dass eine Annahme, die wir den soeben angestellten Betrach- 
tungen zu Grunde gelegt haben, für den Muskel nicht zutrifft. Ich nahm an, der Muskel setze den contractilen Kräften einen Widerstand entgegen, der für ein und denselben Grad von Verkürzung constant sei. Das ist thatsächlich nicht der Fall, der Widerstand ist variabel, das Bemerkenswerthe ist aber, dass die Veränderung des Widerstands bedingt ist durch die Nachschrumpfung: je grösser die Nachschrumpfung desto geringer ist der innere Widerstand. Diese Veränderung des Widerstandes braucht hinsichtlich ihrer Wirkung auf den Zuckungsverlauf nicht getrennt von der Nachschrumpfung betrachtet zu werden aus folgendem Grunde :

Die Nachschrumpfung hat Antheil an der Grösse der gesammten Verkürzung des Muskels dadurch:

1) Dass sie eine Gestaltveränderung hervorruft in gleichem Sinne, wie der physiologische Contractionsact, die sich zu der physiologischen Contraction hinzu gesellt.

2) Dass mit Zunahme der Nachschrumpfung auch die inneren Widerstände abnehmen, die sich den contractilen Kräften entgegenstellen.

Gestaltveränderung durch Nachschrumpfung und Widerstandsänderung wirken also in gleichem Sinne auf die Verkürzung. Die gleiche contractile Kraft bewirkt eine um so grössere Verkuìrung, je grösser die Nachschrumpfung ist.

Wie wirkt nun die Spannungsvermehrung auf die Nachschrumpfung ein? Wir wollen wieder eine Zuckung mit Anfangshemmung vergleichen mit einer ungehemmten Zuckung. Bei ersterer wird die durch den physiologischen Contractionsact allein hervorgebrachte Verkürzung gehemmt bis zum Moment der Entspannung, mithîn müsste auch ihre Folge, die Nachschrumpfung, bis dahin verhindert werden. Die Nachschrumpfung ist also bei der Zuckung mit Anfangshemmung geringer als bei der ungehemmten Zuckung, mithin miüste auch die Verkürzung nach der Entspannung durchweg geringer sein, als bei der isotonischen Zuckung. Diese Ueberlegung führt also zu der Ansicht, die ich schon früher ausgesprochen habe ${ }^{1}$ ), dass „nach $\mathrm{Blix}$ die Spannungszunahme auf die Verkürzung keine andere Wirkung haben" kann, "als eine hemmende, dịe bewirkt wird durch geringere Nachschrumpfung“.

1) Dies Archiv Bd. 59. S. 400. 
Zur Frage: $0 \mathrm{~b}$ d. physiol. Contractionsact von d. Spannung beeinflusst wird. 503

Das Hinausgehen der Verkürzung bei Zuckungen mit Anfangshemmung, bei Schlenderzuckungen, bei Anschlagzuckungen des kalten Muskels über die isotonische wäre demnach durch die Hypothese von $\mathrm{Blix}$ nicht za erklären.

Blix hilft sich in folgender Weise:

1) Er behauptet, dass ein Theil dieser Erscheinungen - nämlich die Zuckungen mit Anfangshemmung und die Scbleuderzuckungen - bedingt seien durch entstellte Wiedergabe der Verkürzung in den Curven in Folge Schleuderung des Längenzeichners.

2) Die Erscheinungen, die auch er nicht durch die Schleuderung des Längenzeichners erklären kann (Anschlagzuckungen), erklärt er, indem er behauptet, vermehrte Spannung könne wohl unter Umständen die Nachschrumpfung vergrössern.

Was zunächst den ersten Pankt anlangt, so muss ich Blix widersprechen. Ich gehe zuerst auf die Versuche mit Schleuderzuckungen von Sogalla ${ }^{1}$ ) ein. Blix behauptet, das Hinausgehen der Schlenderzuckungscurve über die isotonische in diesen Versuchen sei durch Schleuderung des Längenzeichners, also durch ungetreue Wiedergabe der eigentlichen Verkürzung bedingt, denn „der Längenzeichner geht schliesslich viel schneller in die Höhe, als bei der sogenannten isotonischen Zuckungscurve". Die Durchsicht des Versuchsmaterials Sogalla's, das mir hier noch zur Verfuigung stand, sowie meines eigenen Materials hat aber ergeben, dass in solchen Fällen, wo der Längenzeichner nach dem Emporwerfen des Schleuderhebels steiler in die Höhe geht, als in der isotonischen Curve, die Schleuderzuckungscurve immer ganz in das Areal der isotonischen hineinfällt; das Hinausgehen der Sehlenderzuckungscurve über die isotonische kommt nur dann vor, wenn erstere weniger steil ansteigt, als letztere (siehe, Fig. 1, P fl ïg er's Archiv Bd. 61, S. 82).

Schon S og alla hat beobachtet, dass zuweilen der Gipfel der Schleuderzuckungscurve sogar höher lag, als der der isotonischen. Ich habe später ${ }^{2}$ ) die Bedingungen ausfindig zu machen gesucht, die diese Erscheinung begünstigen. Blix führt auch diese Erscheinung auf Schleuderung des Längenzeichners zurück, beachtet dabei aber gar nicht, dass die für sie guinstigen

1) Dissertat. Würzburg 1889.

2) Dies Archiv Bd. 50. S. 166. 
Bedingungen für die Schleuderung des Längenzeichners besonders ungünstig sind, denn die Erscheinung kommt um so leichter zu stande, je niedriger die Temperatur des Muskels ist, je träger also die ganze Zuckung verläuft.

Betreffs des Hinausgehens der Zuckungen mit Anfangshemmung hat schon von $\mathrm{Kr}$ ies ${ }^{1}$ ), der dariiber zuerst Beobachtungen anstellte, die Gründe angeführt, die gegen die Ansicht sprechen, dass es sich hier um Wurf handelt. Ich kann auf die Auseinandersetzungen von $\mathrm{K} \mathrm{r}$ i e s' verweisen, da ich denselben nichts zuzufugen habe.

Die Möglichkeit, dass durch Schleuderung des Längenzeichners eine Entstellung der Curven zu erhalten ist, will ich durchaus nicbt leugnen, in der Mebrzabl der Fälle von Zuckungen mit Anfangshemmung bei von $\mathrm{K}$ ries und von Schleuderzuckungen bei Sog a ll a und mir beruht das Hinansgehen der Zuckungseurve ïber die isotonische aber sicher nicht auf Schleuderung des Längenzeichners.

Da, wo Blix das Hinausgehen der Curve der Zuckung mit Spanuungsänderung über die isotonische selbst nicht durch Schleuderung des Längenzeichners erklären kann, nämlich bei den Anschlagzuckungen des kalten Muskels, hilft er sich mit einer anderen Hypothese. Er behauptet, durch Spannungszunahme könne einer der Vorgänge, die bei der Nachschrumpfung betheiligt seien, verstärkt werden. Es würde also zu einer grösseren Nachschrumpfung: kommen, dadurch wäre die grössere Verkürzung bedingt. Er begründet diese Ansicht etwa so:

"Wenn man einen gespannten, mit beiden Enden fixirten Muskel reizt, so werden freilich bei oberflächlicher Betrachtung die änsseren Conturen des Muskels unverändert erscheinen. Und doch ändern die meisten der Muskelsegmente ihre Form, indem einige sich 'verkürzen, andere gedehnt werden, da die zusammenziehende Kraft nicht überall gleich ist oder nicht in jedem Zeitmomente durchgehend dieselbe Entwicklung erreicht bat". So "entsteht bei der Zuckung eine Verschiebung zwischen den Muskelrohren und Muskelbündeln“. „In dem Momente, da der physiologische Contractionsact überall voruber ist, besteht darum noch eine Verschiebung unter den Muskelsegmenten and es wird eine gewisse

1) a. a. 0. 
Zur Fráge: Ob d. physiol. Contractionsact von d. Spannung beeinflusst wird. 505

Arbeit nöthig, um sie in die Lage zu bringen, die sie vor der Erregung einnahmen." Das Ausführen dieser Arbeit erfordert Zeit." „Dieselbe innere Verschiebung muss, wenn auch in kleinerem Umfange, bei jeder Contraction eintreffen, wahrseheinlich am wenigsten bei geringer Spannung und mehr, je grösser die Spannung im Verlaufe der Contraction gewesen ist".

Dazu habe ich Folgendes zu bemerken. Die Behauptung, dass die erwähnten inneren Verschiebungen am wenigsten bei geringer Spannung vor sich gehen, ist nicht nur ganz unbewiesen, sondern erscheint mir äusserst unwahrscheinlich. Im ungespannten Mugskel finden die ungleichzeitigen und ungleich kräftigen Contractionen verschiedener Segmente gerade so statt, wie im gespannten - weil ja nach Blix der Contractionsact selbst keine Aenderung durch die Spannụng erfährt. Absolut sind die Gestaltveränderungen im ungespannten Muskel aber grösser, als in gespannten und deshalb sollte man meinen, dass die inneren Verschiebungen, die durch die Gestaltveränderungen hervorgebracht werden, im ungespannten Muskel auch grösser seien, als im gespannten.

Aber zugegeben, Blix habe Recht, dann wird man doch immerhin fül wahrscheinlich halten mïssen, dass der Unterschied in der Grösse der inneren Verschiebungen bei ungespanntem und gespanntem Muskel nur ein geringer sein kann. Das Hinausgehen der Anschlagzuckung über die isotonische ist aber oft ein sebr beträchtliches. In dem von Blix Fig. 3. S. 247 angeführten Falle liegt das Endstück der An'schlagzuckung z. B. um einen Betrag höher als die isotonische, der $1 / 3$ der ganzen isotonischen Verkürzung ausmacht. Ich finde in meinen Versuchen oft Fälle, wo eine Scbleuderzuckungscurve um die Hälfte des Betrags der ganzen isotonischen Hubhöhe böher liegt als die isotonische. Das durch . Hemmung der Verktirzung ein Zuwachs an Verkürung auf rein mechanischem Wege, und noch dazu ein so grosser erbalten wird, erscheint mir einfach widersinnig.

Aber es giebt eine Thatsache, durch die die Ansicht B lix' direct widerlegt wird. Die von $B 1$ i $x$ angenommenen Verschiebungen mitssen im Sinne seiner Ansicht am grössten sein bei einer isometrischen Zuckung mit kleiner Anfangsspannung. Denn die ungleichzeitigen und ungleichkräftigen Contractionen in verschiedenen Muskelsegmenten bedingen bei der isometrischen Zuckung in den einen Segmenten Verdickung, in anderen Verdünnung durch 
Dehnung und dadurch die Verschiebungen; die Verdickungen einerseits, die Verdlinnungen anderseits müssen um so geringer sein, je grösser die Anfangsspannung, weil der gleiche Zuwachs an Spannung in den einzelnen Segmenten relativ um so geringer" ist, je grösser die Spannung schon von vorne herein ist. Mithin müssen auch die Verschiebungen um so geringer sein, je grösser die An. fangsspannung. In den Curven von isometrischen Zuckungen mit verschiedener Anfangsspannung müsste dies Verhalten so zum Ausdruck kommen, dass die Curve mit grosser Anfangsspannung: steiler absinkt und schneller die Abscissenachse erreicht als die mit kleiner Anfangsspannung. Thatsächlich ist aber gerade das Umgekehrte der Fall, wie schon Fick friher ${ }^{1)}$ angegeben hat und wie ich auf Grund von Untersuchungen mit dem Schönlein'schen Spannungszeichner bestätigen kann. Ich habe sogar öfter beobachtet, dass die absolute Spannungszunahme in der Gipfelzeit der Zuckungscurve bei der grossen Anfangsspannung kleiner ist, als bei der geringen und dass trotzdem die durch den Contractionsact hervorgebrachte Spannung gegen Ende der Zuckung bei der grossen Anfangsspannung grösser ist als bei der kleinen. Zeichnet man die so erhaltenen Curven über derselben Abscissenachse auf, so liegt die Curve bei grosser Anfangsspannung also mit ihrem Gipfel innerhalb, mit dem Endstiick ausserbalb des Areals der Curve mit kleiner Anfangsspannung.

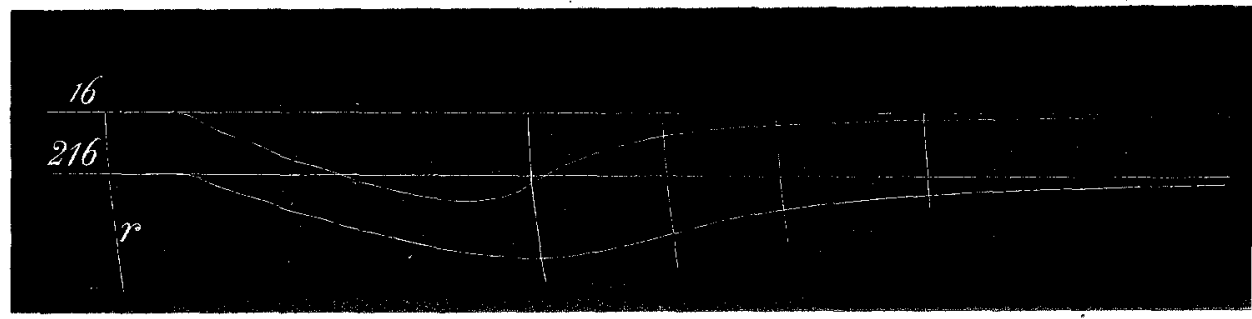

Figur 1.

In der vorstehenden Figur 1 gebe ich Curven wieder, die bei solchen Versuchen erhalten wurden. Als Präparat diente der Froschgastrocnemius, der von seinem Nerven aus maximal gereizt wurde. Das obere Ende war am Spannungszeichner befestigt, das untere festgestellt. Die beiden Anfangsspannungen waren 16 und

1) Mechan. Arbeit u. Wärmeentwickelung etc. Leipzig 1882. S. 132. 
Zur Frage: $0 \mathrm{~b}$ d. physiol. Contractionsact von d. Spannung beeinflusst wird. 50 ?

216. gr; bei den Abscissen sind die zugehörigen Anfangsspannungen angegeben. Zuerst wurde die Curve mit Anfangsspannung $216 \mathrm{gr}$ gezeichnet, dann die mit $16 \mathrm{gr}$. $r$ ist für beide Curven der Reizmoment. Im Abstieg der Curven sind 4 Linien rom Spannungszeichner bei feststehender Schreibtrommel gezeichnet worden, die bei dem Ausmessen der Carven die Berücksichtigung der bogenförmigen und nicht senkrechten Beẉegung der Schreibspitze erleichtern. Die Graduirung des Spannungszeichners hatte ergeben, dass im Bereiche der in unserem Falle vorkommenden Spannungen die Spannung proportional dem Ausschlag der Zeichen. spitze ist, so dass die Höhen beider Curven ohne Umrechnung mit einander verglichen werden können. Man nimmt wahr, dass die Curve von $16 \mathrm{gr}$ schneller absinkt als die von $216 \mathrm{gr}$, dass in letzterer im Abstieg die Spannung durchweg grösser ist, als in ersterer. Die grösste überhaupt erreichte Spannung ist aber bei $216 \mathrm{gr}$ um ein geringes kleiner als bei $16 \mathrm{gr}$.

Auf Grund dieser Ueberlegungen und Beobachtungen muss ich also die Beweisführung B l i x' für misslungen erachten.

Beiläufig sei noch bemerkt, dass B li x meine Erklärung der in Rede stebenden Erscheinungen unrichtig wiedergiebt. Nicht durch eine Verspätung des „zweiten Muskelprocesses“ erkläre ich sie - ich nehme im Gegentheil eine Beschleunigung desselben durch die Spannung an - sondern durch eine Verstärkung des "ersten Processes". Eine Verspätung des "zweiten Muskelprocesses" durch die Spannung nehmen a ${ }^{1}$ ) und Kohnsta m m$^{2}$ ) an, aber, wie ich gezeigt habe ${ }^{3}$ ), ohne zureichenden Grund.

Nun giebt es ausser der eben discutirten fördernden Wirkung der Spannung auf die Verkürzung noch eine hemmende. Diese würde durch die Annahme von B lix erklärlich sein. So sollte man wenigstens auf den ersten Blick hin meinen. Indessen habe ich auch hiergegen Bedenken erhoben. Denn die Hemmung der Verkürzung erschien in einigen Versuchen, die mit Anschlagzuckungen des warmen Muskels angestellt worden waren, so gross, dass die Hypothese B l i x' zur Erklärung nicht ausreichte.

Hiergegen wendet sich nun B lix mit der Behauptnng, meine

1) Verh. d. physiolog. Ges. Berlin 14. Oktober 1892 .

2) D u Bois - Re y mond's Archiv 1893. S. 49.

3) Dies Archiv Bd. 55. S. 175 u. Centralbl. f. Physiol. 1893, Heft 19. 
Beobachtungen beruhten auf Versuchsfehlern. Der frühere Abfall des Endstiicks der Anschlagzuckung habe in Wirklichkeit nicht statt, sondern sei in meinen Versuchen bloss vorgetäuscht gewesen durch die Entstellung der Curven durch Seblenderung des Längenzeichners. Wenn man einen leichteren Hebel verwende erhalte man auch beim warmen Muskel ein Hinausgehen der Anschlagzuckung iiber die isotonische.

Ich habe deshalb meine fruheren Versuche mit Anschlagzuckungen des warmen Muskels wiederholt, und zwar mit Verwendung eines sehr leichten Hebels, der keine in Betracht kommenden Trägheitsschwingungen zeigte. Als Präparate wurden verwendet der Gastrocnemius und Sartorius. Es hat sich nun allerdings ergeben, dass die Verfrülung des Endstïcks der Anschlagzuckung des warmen Muskels nicht so gross ist, als ich nach meinen früheren Versuchen angenommen habe, ja es zeigte sich sehr häufig ein Hinausgehen desselben über die isotonische Curve. Aber dieses Hinausgehen war durchaus nicht so beträchtlich wie man nach den Auseinandersetzungen B li x' erwarten sollte, sondern sehr geling und dann kam es, auch wenn die Anschlagzuckung schliesslich über die isotonische hinausging, meist vor, dass der Abstieg der Anschlagzuckungscurve noch innerhalb des Areals der isotonischen begann. In einigen Fällen trat der Beginn des Abfalls etwa in der Zeit ein, der dem Gipfelpunkt der isotonischen entsprach und für solche Fälle würde meine fribhere Berechnung vollständig zntreffen, einerlei ob die Anschlagzuckung schliesslich noch iber die isotonische hinausgeht oder nicht.

Nachdem freilich B li x erklärt hat, dass er meine Beobachtungen über Anschlagzuckungen des warmen Muskels nicht bestätigen konnte, werde ich mich nicht mebr darauf berufen können, um ihn zu überzeugen. Aber jch komme deshalb nieht in Verlegenheit, weil es noch andere Beobachtungen giebt, für die meine früheren Ausführungen und Berechnungen ganz zutreffen, und bei denen eine Entstellung der Curven durch Trägheit des Längenzeichners genügend ausgeschlossen ist. Unter anderen lassen sich so verwerthen die Resultate der kürzlich von mir beschriebenen Versuche mit "Zugzuckungen " ${ }^{1}$ ), in denen die Spannungsänderung hervorgebracht wurde durch eimen kurz dauernden Zug am unteren

1) Dies Archiv Bd 61. S, 77. 
Zur Frage: Ob d. physiol. Contractionsact von d. Spannung beeinflusst wird. 509

Muskelende. Fig. 6 auf S. 90, Bd. 61 dieses Archives, die ich hier in Fig. 2 nochmals abdrucken lasse, giebt eine so erhaltene Curve wieder. Die Figur enthält ausser der Zugzuckungscurve noch eine isotonische und eine Anschlagzuckungscurve. Der Abstieg der

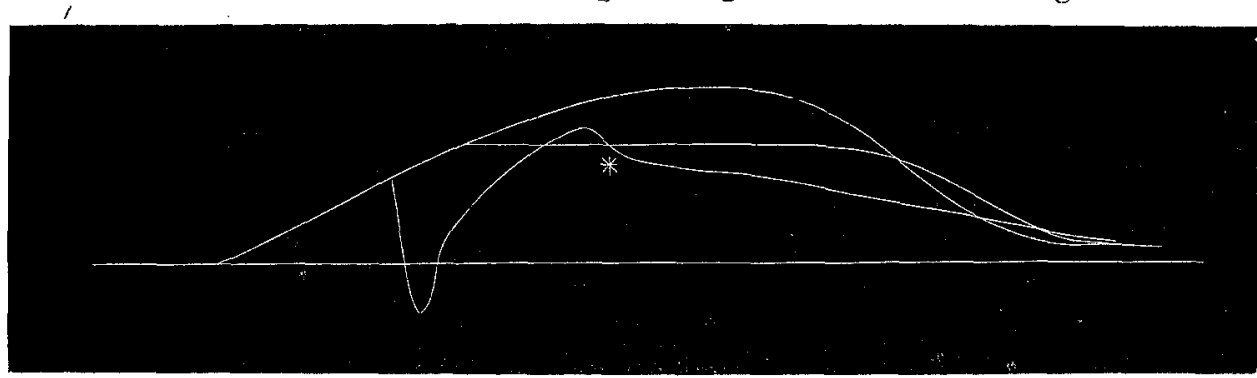

Fig. 2.

Zugzuckungscurve ist verfrült, er liegt fast ganz im Areal der isotonischen bis auf ein kleines Stück am Ende. In der isotonischen Curve findet sich nur am Ende, eine Einbuchtung nach unten, die vielleicht als Schleuderung gedeutet werden könnte, dieselbe ist aber gering. Da der Anstieg der isotonischen Curve noch viel weniger steil als der Abstieg ist, dürfen wir behaupten, dass in ihm eine etwaige Schleuderung zu klein ist, um eine in Betracht kommende Entstellung der Curve in diesem Theile bewirkt zu haben; mithin wird der Gipfel der Curve auch der wirklichen Gipfelzeit der Zuckung entsprechen. In der Curve der Zugzuckung: ist vielleicht der Gipfel entstellt wiedergegeben, weil bei dem rapiden Anstieg nach Aufhören des Zuges Schleuderung eingetreten sein könnte, aber wir dürfen annehmen, dass von dem Punkte * ab die Curve wieder der getreue Ausdruck der Längenänderung des Muskels ist, weil von da ab der Abstieg sogar weniger steil ist, als der Anstieg der isotonischen Curve. Spätestens vom Punkte * ab erfolgt also der Abstieg bei der Zugzuckung. Dieser Punkt liegt aber erheblich früher, als die Gipfelzeit der isotonischen Curve.

Es lässt sich nun hier folgende Betrachtung anknüpfen: In dem Zeitpunkt, der der Gipfelzeit der isotorischen Curve entspricht und der in der Curve $50 \mathrm{~mm}$ vom Beginn der Verkürzung entfernt ist, beträgt die Ordinate für die Zugzuckung $9 \mathrm{~mm}$, für die isotonische $18 \mathrm{~mm}$, in letzterer ist also die Verkürzung doppelt so gross, al's in ersterer. Wie ist diese Thatsache im Sinne der $\mathrm{Hy}$ pothese von B lix zu erklären? Zunächst wird zu erörtern sein, 
ob die Erscheinung zu erklären ist durch die eingangs besprochene Wirkung der inneren Widerstände. Die Entspannung erfolgt bei der Zugzuckung momentan nach dem kurzen Zug, der das untere Muskelende nach unten gezogen und den Längenzeichner in unserem Falle bis unter die Abscissenachse bewegt hat. 1/40" nach der Entspannung muss der Muskel nach dem vorhin Gesagten soweit verkürzt sein, wie in entsprechender Zeit bei Isotonie, falls die Aenderung des Zuckungsverlaufs nur durch jene Wirkung der Widerstände bedingt sein soll. Rechnen wir die ganze Zuckungsdaner zu $1 / 10 "$, so ist der Punkt $1 / 40$ " nach der Entspannung ungefähr zu suchen in dem Punkte ${ }^{*}$ der Zugzuckungscurve. Soweit die Curve von da ab gegen die isotonische verändert erscheint, muss diese Aenderung bedingt sein durch etwas anderes, als jene Wirkung der Widerstände. Auch die Thatsache, dass die Curve der Zugzuckung schon früher abzufallen beginnt, als die isotonische, spricht für diese Ansicht, denn wenn nur die Verzögerung der Verkürzung durch die Widerstände Schuld an dem Verlauf der Zugzuckungscurve wäre, müsste die Curve gerade so lang ansteigen, wie auch die isotonische ansteigt.

Wenn die geringere Höhe der Zugzuckungscurve nicht durch den Einfluss des Widerstands bedingt ist, bleibt im Sinne der Ansicht von Blix nichts anderes iubrig, als die Annahme, dass sie nur beruht auf einer geringeren Betheiligung der Nachschrumpfung an der Verkürzung. Das führt zu dem Schlusse, dass mindestens die Hälfte der ganzen isotonisehen Hubböhe durch Nachschrumpfung zu Stande kommt.

Aber die Nachschrumpfung muss an der isotonischen Hubhöhe noch mehr betheiligt sein, weil ja auch in der Zugzuckung in dem der isotonischen Gipfelzeit entsprechenden Zeitpunkt Nachschrumpfung im Muskel bestehen muss. Denn auch in der Zugzuckung findet ja Verkuirzung dureh contractile Kräfte statt, welche Nachschrumpfung zur Folge haben muss. Wie gross dieser Betrag der Nachschrumpfung ist, lässt sich durch folgende Veberlegung ermitteln: Die Nachschrumpfung wird um so grösser sein, je grösser die durch contractile Kräfte bedingte Contraction ist, und je länger sie anbält. In gleichen Zeiträumen vom Beginn der Verkürzung ab wird die Nachschrumpfung demnach wohl etwa proportional der mittleren Verkürzung sein. Um die mittleren Verkürzungen zu erhalten, messen wir die Flächen aus, die von 
den Curven, der Abscissenachse und den in die Gipfelzeit der isotonischen Curve fallenden Ordinaten begrenzt werden (wobei natürlich das Stïck, dąs bei der Zugzuckung unter der Abscissenachse liegt, negativ zu setzen ist) und dividiren durch das Stück Abscisse, das die Flächen begrenzt. Die Ausmessung der Flächen hat ergeben: Für die isotonische Carve 535 Quadratmillimeter, für die Zugzuckung 321 Quadratmillimeter, die Abscissenlänge beträgt $50 \mathrm{~mm}$, mithin die mittlere Verkürzung für Isotonie: $10,7 \mathrm{~mm}$, für die Zugzuckung 6,42 mm, das Verhältniss der mittleren Verkïrzungen genau wie $5: 3$. Demnach beträgt die Nachschrumpfung bei der Zugzuckung in dem der isotonischen Gipfelzeit entsprechenden Zeitpunkt $8 / 5$ der isotonischen. Setzen wir die gesammte Nachschrumpfung der isotonischen Zuckung gleich $x$, und die isotonische Hubhöhe minus der Höhe der Zugzuckung in entsprechender Zeit, die nach dem vorhin Gesagten in Nachschrumpfung bestehen muss, gleich $a$, so ergiebt sich:

$$
x=\frac{3}{5} x+a \text { oder } x=2,5 \text {. } a .
$$

Da die ganze isotonische Verkürzung gleich $2 a$ ist, so heisst das mit Worten: Im Sinne der Hypothese von Blix würde die gesammte Nachschrumpfung der isotonischen Zackung um $1 / 4$ grösser sein, als die wirkliche isotonische Hubhöhe. Diese Hypothese führt. also zu unmöglichen Consequenzen.

B li $x^{\prime}$ wird mir freilich wohl einwenden, dass ich die Zahlen für ihn zu ungünstig angenommen habe. Denn in der Curve der Zugzuckung könnte möglicherweise Entstellung durch Schleuderung des Längenzeichners enthalten sein, und zwar in dem Stlick, in dem der Zeichner nach der plötzlichen Entspannung schnell nach aufwärts bewegt wird. Aber selbst wenn man dies berücksichtigt und Correctur vornimmt, erhält man immer noch für die mittlere Hubhöhe der Zugzuckung einen Betrag, der noch grösser als die Hälfte der isotonischen mittleren Hubhöhe ist, so dass bei der Berechnung immer noch Unmögliches herauskommt.

Wie der Verlauf der Zuckungscurve im Sinne der Hypothese von Blix sein müsste, lässt sich iibrigens entnehmen aus einer anderen Figur in meiner erwähnten Abhandlung. In Fig. 4 a ist auch eine Zugzuckungscurve enthalten, in der der Zug allerdings so früh beginnt, dass eine merkliche Beeinflussung des Contractionsprocesses im Sinne der Lehre, die ich vertheidige, noch nicht statt haben kann, weil der Contractionprocess bei Beginn des Zuges

E. Pflủger, Archiv für Physiologie. Bd. 62. 
512 F. Schenck: Zur Frage: Ob der physiol. Contractionsact etc.

noch wenig entwickelt war. Hier steigt in Folge Schleuderung des Längenzeichners die Curve steil an und geht weit über die isotonische hinaus, schliesslich aber geht sie nur wenig unter der isotonischen her und fällt am Ende mit ibr zusammen. Da die rein mechanischen Verhältnisse des Muskels in diesem Falle nicht andere sein können, als in dem Falle, wo der Zug etwas später beginnt, so müsste nach $\mathrm{B} 1 \mathrm{ix}$ bei späterem Beginn des Zuges der Verlauf der Zugzuckung ein ganz entsprechender sein. Aber davon ist nichts zu merken; in Fig. 4 b, die von demselben Muskel wie Fig. 4 a bei späterem Beginn des Zuges erhalten ist und die fast gerade so wie die von einem anderen Präparate erhaltene Fig 6 aussieht, erreicht die Zugzucknngscurve nach der Entlastung noch nicht einmal die Höhe der isotonischen und liegt im Uebrigen im Abstieg viel tiefer, als diese.

Aehnliche Betrachtungen lassen sich an die Ergebnisse der Versuche mit „Zugschleuderzuckungen" (Fig. 7 und 8 der erwähnten Abhandlung) anknipfen. Hier ist aus der Fjg. 7, welche die Spannungs- und Längenänderung bei dem Zug mit darauf folgender Schleuderung eines Schwunghebels am ruhenden Muskel wiedergiebt, die Zeit zu entnehmen, die der Muskel braucht, um sich nach der Entspannung zu verkïrzen. Die so bestimmte Zeit kann man benutzen zur Bestimmung des Punktes in der Zugschleuderzuckungscurve Fig. 8, wo der Muskel nach der Entspannung das Maximum der Verkürzung erreicht haben muss. Diese Versuche haben auch den Vortheil, dass die Spannungsänderung mitregistrirt wurde. Auch hier lassen sich die Resultate nicht mit Blix' Hypothese vereinigen.

Ich glaube damit ron neuem bewiesen zu haben, dass $B 1$ i $x^{\prime}$ Hypothese zur Erklärung der Thatsachen unzureichend ist, und dass kein Grund vorliegt, die alte Lehre preiszugeben. 